\title{
AXIAL SYMMETRY AND CLASSIFICATION OF STATIONARY SOLUTIONS OF DOI-ONSAGER EQUATION ON THE SPHERE WITH MAIER-SAUPE POTENTIAL*
}

\author{
HAILIANG $\mathrm{LIU}^{\dagger}$, HUI ZHANG ${ }^{\ddagger}$, AND PINGWEN ZHANG ${ }^{\S}$
}

\begin{abstract}
We study the structure of stationary solutions to the Doi-Onsager equation with Maier-Saupe potential on the sphere, which arises in the modelling of rigid rod-like molecules of polymers. The stationary solutions are shown to be necessarily a set of axially symmetric functions, and a complete classification of parameters for phase transitions to these stationary solutions is obtained. It is shown that the number of stationary solutions hinges on whether the potential intensity crosses two critical values $\alpha_{1} \approx 6.731393$ and $\alpha_{2}=7.5$. Furthermore, we present explicit formulas for all stationary solutions.
\end{abstract}

Key words. Axial symmetry, Stationary solutions, Doi-Onsager model.

AMS subject classifications. 76B03,65M12,35Q35

\section{Introduction}

We begin with the Doi-Onsager model

$$
\frac{\partial f}{\partial t}=D_{r} \mathcal{R} \cdot(\mathcal{R} f+f \mathcal{R} U)
$$

where $f(t, x)$ denotes the orientation distribution function, and $D_{r}$ is the rotational diffusivity, which, without loss of generality, will be set to 1 . Here $x \in \mathbb{S}^{2}, \mathbb{S}^{2}$ is the unit sphere and

$$
\mathcal{R}=x \times \frac{\partial}{\partial x}
$$

is the differential operator on $\mathbb{S}^{2}$. $U$ is the mean-field interaction potential. This equation arises in the modelling of rigid rod-like molecules of polymers. Different expression of potentials leads to different models. Onsager considered the potential

$$
U(x) \triangleq U(x,[f])=\alpha \int_{\left|x^{\prime}\right|=1}\left|x \times x^{\prime}\right| f\left(x^{\prime}\right) d x^{\prime} .
$$

We will consider the Maier-Saupe potential $[4,10]$, defined by

$$
U(x)=\alpha \int_{\left|x^{\prime}\right|=1}\left|x \times x^{\prime}\right|^{2} f\left(x^{\prime}\right) d x^{\prime},
$$

where $\alpha$ is a parameter that measures the potential intensity. From the Doi-Onsager equation (1.1) we see that $\int_{|x|=1} f(t, x) d x$ is conserved. Therefore (1.1) is often solved together with an enforced normalization

$$
\int_{|x|=1} f(x) d x=1
$$

${ }^{*}$ Received: Febuary 10, 2005; accepted (in revised version): April 11, 2005. Communicated by Shi Jin.

${ }^{\dagger}$ Department of Mathematics, Iowa State University, Ames, IA 50011-2064, U.S.A. (hliu@iastate. $\mathrm{edu})$.

${ }^{\ddagger}$ School of Mathematical Sciences, Beijing Normal University, Beijing, 100875, P.R. China (hzhang@bnu.edu.cn).

$\S$ LMAM and School of Mathematical Sciences, Peking University, Beijing, 100871, P.R. China (pzhang@pku.edu.cn). 
This model has a free energy

$$
A(f)=\int_{|x|=1}\left[f(x) \ln f(x)+\frac{1}{2} f(x) U(x)\right] d x,
$$

as its Lyapunov functional. We will be interested in the stationary solutions of (1.1):

$$
\mathcal{R} \cdot(\mathcal{R} f+f \mathcal{R} U)=0
$$

The Doi-Onsager equation for rod-like molecules has been very successful in describing the properties of liquid crystalline polymers in a solvent, see e.g., $[4,8,9,10,14,15]$. The basic object in the Doi-Onsager equation is the single molecule position-orientation distribution function. Interactions between molecules are modelled by a mean-field potential. Therefore, the Doi-Onsager equation can be regarded as a mean-field kinetic theory. Besides interaction with other rods, the rods are also interacting with the flow and are subject to Brownian forces. If the interaction strength is sufficiently strong, compared with the Brownian forces, or if the rod concentration is sufficiently high, then the system prefers to be in a nematic phase in which the rods tend to line up with each other. Otherwise the system is in an isotropic phase in which the orientation of the rods is completely random.

The Doi-Onsager equation is known to exhibit nontrivial nonlinear features, e.g., $[1,11]$ and its study has recently attracted great attention, e.g., $[8,14,15]$. The general Doi-Onsager equation describes the rotation and translation of polymeric molecules convected with the flow. A basic feature of this model is its ability to describe both the isotropic and nematic phases, e.g., $[4,10,16]$. The complex dynamical properties are amplified considerably when the phase changes. Such a remarkable phase transition phenomenon in rigid rod-like polymers has been observed in both experiments and numerical simulations, e.g., $[8,9,14,15]$.

In this paper we focus our attention on the Doi-Onsager equation with the MaierSaupe potential (1.4). We study structure and phase transitions to stationary solutions of the Doi-Onsager equation on the sphere. Such a phase transition problem was first described by Onsager in 1949 [16], using a variational approach. He used the free energy (1.6) with the potential (1.3), by restricting $f$ to be of the form

$$
f(x)=\frac{\beta}{4 \pi \sinh \beta} \cosh (\beta x \cdot y)
$$

where $y \in \mathbb{S}^{2}$ is a director parameter. $\beta$ is a parameter to be determined from the condition that the free energy be minimized. Here $\beta$ represents the degree of ordering: $\beta=0$ corresponds to the isotropic state where $f=$ const, and $\beta=\infty$ the completely ordered state where $f$ is a Delta function. Onsager was able to argue that in the limit of high concentration one has a transition from the isotropic uniform distribution to an ordered prolate distribution $[1,16]$.

In the last few years, the Doi-Onsager model has attracted a great deal of attention in the mathematics community $[1,2,3,6,13]$. In particular, concerning the structure of stationary solutions of the Doi-Onsager model, Constantin et al. [1] reduced the Doi-Onsager model (1.7) into the nonlinear equations with two parameters and classified these solutions in the high concentration limit. They also proved that the isotropic state is the only possible solution at low enough concentration. The situation is much better understood in the two-dimensional case when the orientation variable lives on the circle. Constantin et al. [2] established a bound on the number 
of stationary solutions, and at the same time, gave a sharp estimate on the region of stability for the isotropic solution. Luo et al. [13] gave a detailed study of the structure of the stationary solutions, proving that there are only two possible solutions, one corresponding to isotropic state, the other corresponding to the nematic state. Their proof was further simplified in $[3,6]$.

For the Doi-Onsager model on the sphere with the Maier-Saupe potential, our main results are: (1) We prove that all stationary solutions are axially symmetric. (2) We give estimates on the sharp characterization of the bifurcation regimes for the isotropic and nematic solutions. Taken together, these results give rather complete understanding of the Doi-Onsager model on the sphere with Maier-Saupe potential.

A different proof of the axial symmetry was done independently and at about the same time by Fatkullin and Slastikov [7].

Now we state our main results. Our first result is about the axial symmetry and explicit representations of the thermodynamic potential $U$.

THEOREM 1.1. Consider the Doi-Onsager equation (1.7) with the normalization (1.5). Let $U$ be the potential defined by (1.4), then such a potential is necessarily invariant with respect to rotations around a director $y \in \mathbb{S}^{2}$, i.e., it is axially symmetric. Moreover, this potential must have the following form

$$
U=\frac{2 \alpha}{3}-\eta\left(|x \times y|^{2}-\frac{2}{3}\right)
$$

where $\eta \in \mathbb{R}$ is a parameter.

We now state our second result on critical intensities of phase transitions and all explicit stationary distributions.

THEOREM 1.2. The number of stationary solutions of the Doi-Onsager equation on the sphere (1.7) with (1.4), (1.5) hinges on whether the intensity $\alpha$ crosses two critical values: $\alpha^{*} \approx 6.731393$ and 7.5 , where

$$
\alpha^{*}=\min _{\eta} \frac{\int_{0}^{1} e^{-\eta z^{2}} d z}{\int_{0}^{1}\left(z^{2}-z^{4}\right) e^{-\eta z^{2}} d z} .
$$

All solutions are given explicitly by

$$
f=k e^{-\eta(x \cdot y)^{2}},
$$

where $y \in \mathbb{S}^{2}$ is a parameter, $\eta=\eta(\alpha)$ and $k=\left[4 \pi \int_{0}^{1} e^{-\eta z^{2}} d z\right]^{-1}$ are determined by $\alpha$ through

$$
\frac{3 e^{-\eta}}{\int_{0}^{1} e^{-\eta z^{2}} d z}-\left(3-2 \eta+\frac{4 \eta^{2}}{\alpha}\right)=0 .
$$

More precisely,

(i). If $0<\alpha<\alpha^{*}$, there exists one solution $f_{0}=1 / 4 \pi$.

(ii). If $\alpha=\alpha^{*}$, there exist two distinct solutions $f_{0}=1 / 4 \pi$ and $f_{1}=$ $k_{1} e^{-\eta_{1}(x \cdot y)^{2}}, \eta_{1}<0$.

(iii). If $\alpha^{*}<\alpha<7.5$, there exist three distinct solutions $f_{0}=1 / 4 \pi$ and $f_{i}=$ $k_{i} e^{-\eta_{i}(x \cdot y)^{2}}, \eta_{i}<0 \quad(i=1,2)$. 
(iv). If $\alpha=7.5$, there exist two distinct solutions $f_{0}=1 / 4 \pi$ and $f_{1}=$ $k_{1} e^{-\eta_{1}(x \cdot y)^{2}}, \eta_{1}<0$.

(v). If $\alpha>7.5$, there exist three distinct solutions $f_{0}=1 / 4 \pi$ and $f_{i}=$ $k_{i} e^{-\eta_{i}(x \cdot y)^{2}}(i=1,2), \eta_{1}<0, \eta_{2}>0$.

Regarding this result several remarks are in order.

1). As for the critical value $\alpha^{*}$, a simple numerical simulation, based on Matlab, of (1.8) gives the result, our numerical calculation indicates that $\alpha^{*} \approx 6.731393$ is accurate up to $10^{-6}$.

2). Our results provide not only a complete picture of phase transitions but also explicit expressions for all stationary solutions. In Theorem 1.2 any stationary solution $f(x)$ with all solutions of the form $f(T x)$, where $T$ is an arbitrary rotation in $\mathbb{R}^{3}$, has been counted as one distinct solution. The identity of each individual function is made by the use of the parameter $y \in \mathbb{S}^{2}$.

3). We should point out that not all stationary solutions are stable. The stability analysis of these solutions is desirable and is under our current study.

4). In physical terms, an isotropic phase corresponds to the case when the distribution function $f=1 / 4 \pi$ and a nematic phase corresponds to the case when $f$ is concentrated at some particular director, which includes the prolate and oblate states. For example, in case $(v)$ of Theorem $1.2, f_{0}=\frac{1}{4 \pi}$ is an isotropic distribution; while the distribution function $f_{1}=k_{1} e^{-\eta_{1}(x \cdot y)^{2}}\left(\eta_{1}<0\right)$ is concentrated in the direction $\pm y$ (called prolate state) and $f_{2}=k_{2} e^{-\eta_{2}(x \cdot y)^{2}}\left(\eta_{2}>0\right)$ is concentrated on the equator perpendicular to $y$ (calledoblate state).

The difficulty of the problem lies in the nonlocal coupling between the potential $U$ and the distribution $f$. The observation [2] of the decoupled equation for $U$ is one of key steps to our approach. The advantage of rewriting a nonlocal equation into a coupled system has been well exploited in different contexts, see [2, 5, 12]. From the decoupled linear equation for $U$, we are able to write out all possible solutions. Some irrelevant solutions are further excluded by using the nonlocal constraint. This enables us to conclude that all potentials must be axially symmetric. We further give explicit expressions for all stationary solutions. Equipped with the solution formula, the determination of the number of solutions reduces to the determination of zeros of a simple function involving a parameter $\eta$ and the intensity $\alpha$. The detailed analysis of the number of zeros of this function in terms of $\alpha$ constitutes another main technical part of this work.

This paper is organized as follows: in Sec. 2, we give a rehearsal of our novel approach applied to the reduced model on a circle. This approach leads to the simplest proof of the result recently obtained in $[3,6,13]$. Sec. 3 is devoted to the analysis of the Doi-Onsager model on the sphere, which is far less trivial than the reduced one. The presentation is split into several lemmas, from which a complete picture on the Doi-Onsager model is naturally shaped.

\section{The Doi-Onsager equation on the circle}

For the two-dimensional case, the Doi-Onsager equation (1.7) with (1.2),(1.4) reduces to the following,

$$
\begin{aligned}
& f_{\theta \theta}+\left(f U_{\theta}\right)_{\theta}=0, \quad \theta \in[0,2 \pi], \\
& U(\theta)=\alpha \int_{0}^{2 \pi} \sin ^{2}\left(\theta-\theta^{\prime}\right) f\left(\theta^{\prime}\right) d \theta^{\prime},
\end{aligned}
$$


subject to the normalization

$$
\int_{0}^{2 \pi} f(\theta) d \theta=1 .
$$

The structure of stationary solutions has been studied in [13], somehow we were unable to extend the approach introduced in [13] to the Doi-Onsager equation on the sphere. The novel approach taken in this paper works for both cases, and particularly provides a simpler proof for the two-dimensional model.

In order to highlight our approach, we now start with the Eq. (2.1)-(2.3). It follows from (2.2) that

$$
U_{\theta}=\alpha \int_{0}^{2 \pi} \sin 2\left(\theta-\theta^{\prime}\right) f\left(\theta^{\prime}\right) d \theta^{\prime}
$$

Further

$$
\begin{aligned}
U_{\theta \theta} & =2 \alpha \int_{0}^{2 \pi} \cos 2\left(\theta-\theta^{\prime}\right) f\left(\theta^{\prime}\right) d \theta^{\prime} \\
& =2 \alpha \int_{0}^{2 \pi}\left[1-2 \sin ^{2}\left(\theta-\theta^{\prime}\right)\right] f\left(\theta^{\prime}\right) d \theta^{\prime} \\
& =2 \alpha-4 U,
\end{aligned}
$$

where (2.3) has been used. Therefore we obtain a decoupled equation (2.4) for $U$

$$
U_{\theta \theta}+4 U=2 \alpha .
$$

Its general solution is

$$
U=\frac{\alpha}{2}+\eta \cos 2\left(\theta-\theta_{0}\right),
$$

where $\eta$ and $\theta_{0}$ are two arbitrary parameters. Without loss of generality we assume $\eta \geq 0$ because a sign change can be always made by simply shifting $\theta_{0}$ to $\theta_{0}+\pi$. With this explicit formula $U$ at hand, we proceed to solve $f$ in terms of $U$. Integration of the equation (2.1) from 0 to $2 \pi$ yields

$$
f_{\theta}+f U_{\theta}=C_{1} .
$$

The integral constant $C_{1}$ has to be zero, as evidenced by the following calculation: integration of (2.6) from 0 to $2 \pi$ gives

$$
\begin{aligned}
2 \pi C_{1} & =\int_{0}^{2 \pi} f U_{\theta} d \theta=\alpha \int_{0}^{2 \pi} \int_{0}^{2 \pi} \sin 2\left(\theta-\theta^{\prime}\right) f(\theta) f\left(\theta^{\prime}\right) d \theta d \theta^{\prime} \\
& =\alpha \int_{0}^{2 \pi} \int_{0}^{2 \pi}\left[\sin 2 \theta \cos 2 \theta^{\prime}-\cos 2 \theta \sin 2 \theta^{\prime}\right] f(\theta) f\left(\theta^{\prime}\right) d \theta d \theta^{\prime}=0 .
\end{aligned}
$$

From (2.6) it follows that

$$
f=C_{2} e^{-U}
$$

Note that the fact $C_{1}=0$ shows the equivalence of the Doi-Onsager equation to the Euler-Lagrange equation obtained from minimizing the free energy (1.6). Using (2.5) we have

$$
f=C e^{-\eta \cos 2\left(\theta-\theta_{0}\right)},
$$


where $C=C_{2} e^{-\alpha / 2}$ is still an arbitrary parameter. The use of relation (2.3) gives

$$
C(\eta)=\left[\int_{0}^{2 \pi} e^{-\eta \cos 2 \theta} d \theta\right]^{-1}
$$

Combining this with the nonlocal constraint (2.2) we obtain the following relation

$$
\frac{\int_{0}^{2 \pi} \sin ^{2}\left(\theta-\theta^{\prime}\right) e^{-\eta \cos 2\left(\theta^{\prime}-\theta_{0}\right)} d \theta^{\prime}}{\int_{0}^{2 \pi} e^{-\eta \cos 2\left(\theta-\theta_{0}\right)} d \theta}=\frac{1}{2}+\frac{\eta}{\alpha} \cos 2\left(\theta-\theta_{0}\right),
$$

which can be further simplified as

$$
\frac{\int_{0}^{2 \pi} \cos 2 \theta e^{-\eta \cos 2 \theta} d \theta}{\int_{0}^{2 \pi} e^{-\eta \cos 2 \theta} d \theta}+\frac{2 \eta}{\alpha}=0 .
$$

It is now clear that the determination of the number of solutions $f$ is equivalent to the determination of the number of $\eta$ in terms $\alpha$ since $C$ is uniquely determined by $\eta$ from (2.9). Therefore we just need to determine the number of zeros of $B(\eta, \alpha)$, which is defined by

$$
B(\eta, \alpha)=\frac{\int_{0}^{2 \pi} \cos 2 \theta e^{-\eta \cos 2 \theta} d \theta}{\int_{0}^{2 \pi} e^{-\eta \cos 2 \theta} d \theta}+\frac{2 \eta}{\alpha} .
$$

We now discuss the critical values of $\alpha$ at which the number of zeros of $B$ changes. First we note that $\eta=0$ is always a zero of $B$ for any $\alpha$. In this case $f=1 / 2 \pi$. Note that $\left|B(\eta, \alpha)-\frac{2 \eta}{\alpha}\right| \leq 1$, which implies that $B(\eta, \alpha)$ becomes positive for large $\eta>0$. It remains to show whether there is another finite zero $\eta^{*}$.

Let $\eta^{*}$ be a zero of $B$, we have

$$
\frac{\int_{0}^{2 \pi} \cos 2 \theta e^{-\eta^{*} \cos 2 \theta} d \theta}{\int_{0}^{2 \pi} e^{-\eta^{*} \cos 2 \theta} d \theta}=-\frac{2 \eta^{*}}{\alpha} .
$$

From (2.12) and using (2.13) it follows

$$
\begin{aligned}
B_{\eta}\left(\eta^{*}, \alpha\right) & =\frac{\left(\int_{0}^{2 \pi} \cos 2 \theta e^{-\eta^{*} \cos 2 \theta} d \theta\right)^{2}}{\left(\int_{0}^{2 \pi} e^{-\eta^{*} \cos 2 \theta} d \theta\right)^{2}}-\frac{\int_{0}^{2 \pi} \cos ^{2} 2 \theta e^{-\eta^{*} \cos 2 \theta} d \theta}{\int_{0}^{2 \pi} e^{-\eta^{*} \cos 2 \theta} d \theta}+\frac{2}{\alpha} \\
& =\frac{4 \eta^{* 2}}{\alpha^{2}}-\frac{1}{2}-\frac{1}{2} \frac{\int_{0}^{2 \pi} \cos 4 \theta e^{-\eta^{*} \cos 2 \theta} d \theta}{\int_{0}^{2 \pi} e^{-\eta^{*} \cos 2 \theta} d \theta}+\frac{2}{\alpha}
\end{aligned}
$$

For $\eta^{*}=0$, we have

$$
B_{\eta}(0, \alpha)=\frac{4-\alpha}{2 \alpha}
$$

For $\eta^{*} \neq 0$,

$$
\begin{aligned}
\int_{0}^{2 \pi} \cos 2 \theta e^{-\eta^{*} \cos 2 \theta} d \theta & =-\eta^{*} \int_{0}^{2 \pi} \sin ^{2} 2 \theta e^{-\eta^{*} \cos 2 \theta} d \theta \\
& =\frac{\eta^{*}}{2} \int_{0}^{2 \pi}(\cos 4 \theta-1) e^{-\eta^{*} \cos 2 \theta} d \theta
\end{aligned}
$$


which combined with (2.13) gives

$$
\int_{0}^{2 \pi} \cos 4 \theta e^{-\eta^{*} \cos 2 \theta} d \theta=\frac{1}{C\left(\eta^{*}\right)}-\frac{2}{\eta^{*}} \frac{2 \eta^{*}}{\alpha C\left(\eta^{*}\right)}=\frac{\alpha-4}{\alpha C\left(\eta^{*}\right)} .
$$

Therefore,

$$
B_{\eta}\left(\eta^{*}, \alpha\right)=\frac{1}{\alpha^{2}}\left[\alpha(4-\alpha)+4 \eta^{* 2}\right] .
$$

From (2.14) and (2.15) we see that there are three cases for $\alpha$ to be distinguished.

(i). If $\alpha<4, B_{\eta}(0, \alpha)>0$. This indicates that $B_{\eta}\left(\eta_{1}, \alpha\right)$ has to be non-positive if $\eta_{1}>0$ is a neighboring zero of $B(\eta, \alpha)$, which contradicts to (2.15). Therefore, in this case there is no zero of $B$ on $(0, \infty)$.

(ii). If $\alpha=4$, then $B_{\eta}(0, \alpha)=0$. A careful calculation gives

$$
B_{\eta \eta}(0, \alpha)=0 \quad \text { and } \quad B_{\eta \eta \eta}(0, \alpha)=\pi^{2}-\frac{\pi}{4}>0,
$$

which implies that $B(\eta, \alpha)>0$ for $0<\eta<\delta$ (small $\delta)$. This fact combined with $B(M, \alpha)>0$ (large $M>0)$ and $B_{\eta}\left(\eta^{*}, \alpha\right)=\frac{\eta^{* 2}}{4}>0$ tells that no such $\eta^{*}>0$ exists.

(iii). If $\alpha>4, B_{\eta}(0, \alpha)<0$, there exists at least one zero for $B$ in $(0, \infty)$ since $B(\infty, \alpha)>0$. On the other hand, the relation $B_{\eta}\left(\eta^{*}, \alpha\right)=\frac{4}{\alpha^{2}}\left(\eta^{*}+\frac{\alpha}{2} \sqrt{1-4 / \alpha}\right)\left(\eta^{*}-\right.$ $\left.\frac{\alpha}{2} \sqrt{1-4 / \alpha}\right)$ implies that there exists at most one zero $\eta^{*}>\frac{\alpha}{2} \sqrt{1-4 / \alpha}$.

The above analysis enables us to conclude the following.

THEOREM 2.1. The number of stationary solutions to (2.1)-(2.3) depends on whether the intensity crosses the critical value $\alpha=4$. More precisely,

(i) If $\alpha \leq 4$, then the only stationary solution is the constant $f=1 / 2 \pi$.

(ii) If $\alpha>4$, then besides the constant solution $f=1 / 2 \pi$, all other stationary solutions are given by

$$
f(\theta)=\frac{e^{-\eta^{*} \cos 2\left(\theta-\theta_{0}\right)}}{\int_{0}^{2 \pi} e^{-\eta^{*} \cos 2 \theta} d \theta}
$$

where $\theta_{0}$ is arbitrary, $\eta^{*}>\frac{\alpha}{2} \sqrt{1-4 / \alpha}$ are uniquely determined by

$$
\frac{\int_{0}^{2 \pi} \cos 2 \theta e^{-\eta^{*} \cos 2 \theta} d \theta}{\int_{0}^{2 \pi} e^{-\eta^{*} \cos 2 \theta} d \theta}+\frac{2 \eta^{*}}{\alpha}=0 .
$$

\section{The Doi-Onsager equation on the sphere}

This section is devoted to the analysis of the three-dimensional case. Consider the Doi-Onsager equation on the sphere with Maier-Saupe potential:

$$
\begin{aligned}
& \mathcal{R} \cdot \mathcal{R} f+\mathcal{R} \cdot(f \mathcal{R} U)=0, \quad x \in \mathbb{S}^{2}, \\
& U(x)=\alpha \int_{\left|x^{\prime}\right|=1}\left|x \times x^{\prime}\right|^{2} f\left(x^{\prime}\right) d x^{\prime},
\end{aligned}
$$

with the normalization

$$
\int_{|x|=1} f(x) d x=1
$$


The proof of Theorem 1.1 and 1.2 will be completed through a series of lemmas. LEMMA 3.1. The solution of (3.1) can be expressed as

$$
f=C e^{-U}
$$

where $C=\left[\int_{|x|=1} e^{-U} d x\right]^{-1}$.

(3.4) can be obtained using a similar argument to (2.7) as in Sec. 2. This is consistent with the Euler-Lagrange equation

$$
\ln f(x)+U(x)=\text { const. }
$$

In order to identify all solutions of $f$, we just need to find all solutions of $U$. To do so, we first obtain a decoupled linear equation for $U$.

Lemma 3.2. The mean-field interaction potential $U$ defined in (3.2) satisfies a decoupled equation

$$
\mathcal{R} \cdot \mathcal{R} U+6 U=4 \alpha .
$$

Proof. Now we change the form of $U$

$$
\begin{aligned}
U & =\alpha \int_{\left|x^{\prime}\right|=1}\left[1-\left(x \cdot x^{\prime}\right)^{2}\right] f\left(x^{\prime}\right) d x^{\prime} \\
& =\alpha-\alpha \int_{\left|x^{\prime}\right|=1}\left(x \cdot x^{\prime}\right)^{2} f\left(x^{\prime}\right) d x^{\prime},
\end{aligned}
$$

where (3.3) has been used. From (3.6) and (1.2) we know

$$
\mathcal{R} U=-2 \alpha \int_{\left|x^{\prime}\right|=1} x^{T} x^{\prime}\left(x \times x^{\prime}\right) f\left(x^{\prime}\right) d x^{\prime}
$$

Therefore,

$$
\begin{aligned}
\mathcal{R} \cdot \mathcal{R} U & =-2 \alpha \int_{\left|x^{\prime}\right|=1}\left(x \times \frac{\partial}{\partial x}\right) \cdot\left[x^{T} x^{\prime}\left(x \times x^{\prime}\right)\right] f\left(x^{\prime}\right) d x^{\prime} \\
& =-2 \alpha\left[\int_{\left|x^{\prime}\right|=1}\left|x \times x^{\prime}\right|^{2} f\left(x^{\prime}\right) d x^{\prime}-2 \int_{\left|x^{\prime}\right|=1}\left(x \cdot x^{\prime}\right)^{2} f\left(x^{\prime}\right) d x^{\prime}\right] \\
& =4 \alpha-6 U .
\end{aligned}
$$

This is the desired equation (3.5).

The potential $U$ also has the following important properties.

LEMMA 3.3. $U(x)$ is a solution of (3.2) if and only if it satisfies

$$
U(x)=\alpha \int_{\left|x^{\prime}\right|=1}\left|x \times x^{\prime}\right|^{2} e^{-U\left(x^{\prime}\right)} d x^{\prime}\left[\int_{\left|x^{\prime}\right|=1} e^{-U\left(x^{\prime}\right)} d x^{\prime}\right]^{-1} .
$$

Moreover, if $U(x)$ is a solution to (3.8), $U(T x)$ is also a solution, where $T$ is an arbitrary rotation operator in $\mathbb{R}^{3}$. 
Proof. The formula (3.4) combined with (3.2) and (3.3) gives (3.8). Let $T$ be an arbitrary rotation operator in $\mathbb{R}^{3}$, then

$$
\begin{aligned}
U(T x) & =\alpha \int_{\left|x^{\prime}\right|=1}\left|T x \times x^{\prime}\right|^{2} e^{-U\left(x^{\prime}\right)} d x^{\prime}\left[\int_{\left|x^{\prime}\right|=1} e^{-U\left(x^{\prime}\right)} d x^{\prime}\right]^{-1} \\
& =\alpha \int_{\left|x^{\prime}\right|=1}\left|x \times T^{*} x^{\prime}\right|^{2} e^{-U\left(x^{\prime}\right)} d x^{\prime}\left[\int_{\left|x^{\prime}\right|=1} e^{-U\left(x^{\prime}\right)} d x^{\prime}\right]^{-1} \\
& =\alpha \int_{\left|y^{\prime}\right|=1}\left|x \times y^{\prime}\right|^{2} e^{-U\left(T y^{\prime}\right)} d y^{\prime}\left[\int_{\left|y^{\prime}\right|=1} e^{-U\left(T y^{\prime}\right)} d y^{\prime}\right]^{-1},
\end{aligned}
$$

where $y^{\prime}=T^{*} x^{\prime}$ has been taken, where $T^{*}$ is the transport of $T$. This implies that $U(T x)$ is also a solution of $(3.8)$.

Based on this observation we are able to prove the following key lemma.

LEMMA 3.4. $U$ is a solution of (3.8) if and only if it can be expressed as

$$
U=\frac{2 \alpha}{3}-\eta\left(|x \times y|^{2}-\frac{2}{3}\right)
$$

where $\eta \in \mathbb{R}, y \in \mathbb{S}^{2}$ are parameters.

Proof. First it is easy to verify $U=2 \alpha / 3$ is a particular solution of (3.5). In order to obtain the general solution of (3.5) we only need to solve the linear homogenous equation

$$
\mathcal{R} \cdot \mathcal{R} V+6 V=0
$$

where $V=U-\frac{2 \alpha}{3}$. Since $\mathcal{R} \cdot \mathcal{R}$ is the Laplace-Beltrami operator on the sphere, its characteristic element functions are the spherical harmonics. The space spanned by the characteristic functions with the characteristic value -6 has five dimensions. Now we write the spherical harmonics in spherical coordinates by setting

$$
x(\theta, \varphi)=\left(x_{1}, x_{2}, x_{3}\right)=(\cos \varphi \sin \theta, \sin \varphi \sin \theta, \cos \theta),
$$

as follows

$$
\begin{aligned}
& Y_{2}^{-2}(\theta, \varphi)=\frac{1}{4} \sqrt{\frac{15}{2 \pi}} \sin ^{2} \theta e^{-2 i \varphi}, \\
& Y_{2}^{-1}(\theta, \varphi)=\frac{1}{2} \sqrt{\frac{15}{2 \pi}} \sin \theta \cos \theta e^{-i \varphi}, \\
& Y_{2}^{0}(\theta, \varphi)=\frac{1}{4} \sqrt{\frac{5}{\pi}}\left(3 \cos ^{2} \theta-1\right), \\
& Y_{2}^{1}(\theta, \varphi)=-\frac{1}{2} \sqrt{\frac{15}{2 \pi}} \sin \theta \cos \theta e^{i \varphi}, \\
& Y_{2}^{2}(\theta, \varphi)=\frac{1}{4} \sqrt{\frac{15}{2 \pi}} \sin ^{2} \theta e^{2 i \varphi} .
\end{aligned}
$$

Therefore the five real linearly independent solutions when written in Eulerian coor- 
dinates are

$$
\begin{aligned}
& Y_{1}=\frac{1}{2}\left(Y_{2}^{-2}+Y_{2}^{2}\right)=\frac{1}{4} \sqrt{\frac{15}{2 \pi}}\left(x_{1}^{2}-x_{2}^{2}\right), \\
& Y_{2}=\frac{1}{2 i}\left(Y_{2}^{2}-Y_{2}^{-2}\right)=\frac{1}{4} \sqrt{\frac{15}{2 \pi}} x_{1} x_{2}, \\
& Y_{3}=\frac{1}{2}\left(Y_{2}^{-1}+Y_{2}^{1}\right)=\frac{1}{2} \sqrt{\frac{15}{2 \pi}} x_{1} x_{3}, \\
& Y_{4}=\frac{1}{2}\left(Y_{2}^{-1}-Y_{2}^{1}\right)=\frac{1}{2} \sqrt{\frac{15}{2 \pi}} x_{2} x_{3}, \\
& Y_{5}=Y_{2}^{0}=\frac{3}{4} \sqrt{\frac{5}{\pi}}\left(x_{3}^{2}-\frac{1}{3}\right) .
\end{aligned}
$$

The general solution $V$ of (3.10) is in the following space

$$
\mathbf{Y} \triangleq \operatorname{span}\left\{x_{1}^{2}-x_{2}^{2}, x_{1} x_{2}, x_{2} x_{3}, x_{1} x_{3}, x_{3}^{2}-1 / 3\right\}
$$

Insertion of $V=U-2 \alpha / 3$ into (3.8) gives

$$
V(x)=\alpha \int_{\left|x^{\prime}\right|=1}\left[\left|x \times x^{\prime}\right|^{2}-\frac{2}{3}\right] e^{-V\left(x^{\prime}\right)} d x^{\prime}\left[\int_{\left|x^{\prime}\right|=1} e^{-V\left(x^{\prime}\right)} d x^{\prime}\right]^{-1} .
$$

Using the rotational invariance we can always choose an operator $T$ such that (3.13) can be simplified as

$$
\begin{aligned}
V(T x) & =\alpha \int_{\left|x^{\prime}\right|=1}\left[\left|T x \times x^{\prime}\right|^{2}-\frac{2}{3}\right] e^{-V\left(x^{\prime}\right)} d x^{\prime}\left[\int_{\left|x^{\prime}\right|=1} e^{-V\left(x^{\prime}\right)} d x^{\prime}\right]^{-1} \\
& =\alpha \int_{\left|x^{\prime}\right|=1}\left[\frac{1}{3}-\left(T x \cdot x^{\prime}\right)^{2}\right] e^{-V\left(x^{\prime}\right)} d x^{\prime}\left[\int_{\left|x^{\prime}\right|=1} e^{-V\left(x^{\prime}\right)} d x^{\prime}\right]^{-1} \\
& =\alpha\left(\frac{1}{3}-\sum_{i=1}^{3} q_{i} x_{i}^{2}\right)
\end{aligned}
$$

where

$$
q_{i} \triangleq q_{i}(V)=\int_{|x|=1} x_{i}^{2} e^{-V(x)} d x\left[\int_{|x|=1} e^{-V(x)} d x\right]^{-1}
$$

is a functional of $V$, up to a rotation. From (3.15) it follows

$$
\sum_{i=1}^{3} q_{i}=1 \quad \text { and } \quad q_{i} \geq 0
$$

Under the operator $T, V(T x)$ is still in $\mathbf{Y}$. We now construct $V(x)$ of the form (3.14) from a larger class (3.12). From (3.12) it follows

$$
V(x)=a_{1}\left(x_{1}^{2}-x_{2}^{2}\right)+a_{2} x_{1} x_{2}+a_{3} x_{1} x_{3}+a_{4} x_{2} x_{3}+a_{5}\left(x_{3}^{2}-1 / 3\right)
$$


with $a_{1}, a_{2}, a_{3}, a_{4}, a_{5}$ to be determined by

$$
V(x)=\alpha\left(\frac{1}{3}-\sum_{i=1}^{3} q_{i} x_{i}^{2}\right) \quad \text { for } \quad \forall x \in \mathbb{S}^{2} .
$$

Setting $x=(0,0,1)$ in $(3.17)$ with $(3.18)$ we obtain

$$
\alpha\left(\frac{1}{3}-q_{3}\right)=\frac{2 a_{5}}{3} .
$$

Setting $x=\left(\frac{1}{\sqrt{2}}, \frac{1}{\sqrt{2}}, 0\right),(1,0,0)$ and $(0,1,0)$, we have

$$
\begin{aligned}
\frac{a_{2}}{2}-\frac{a_{5}}{3} & =\alpha\left(\frac{1}{3}-\frac{1}{2}\left(q_{1}+q_{2}\right)\right), \\
a_{1}-\frac{a_{5}}{3} & =\alpha\left(\frac{1}{3}-q_{1}\right) \\
-a_{1}-\frac{a_{5}}{3} & =\alpha\left(\frac{1}{3}-q_{2}\right)
\end{aligned}
$$

respectively. These relations lead to $a_{2}=0$. Similarly we can show that $a_{3}=a_{4}=0$. Thus (3.17) and (3.18) reduce to

$$
a_{1}\left(x_{1}^{2}-x_{2}^{2}\right)+a_{5}\left(x_{3}^{2}-\frac{1}{3}\right)=\alpha\left(\frac{1}{3}-\sum_{i=1}^{3} q_{i} x_{i}^{2}\right) .
$$

From (3.19), (3.21) and (3.22) it follows that

$$
\begin{aligned}
& \alpha\left(q_{2}-q_{1}\right)-2 a_{1}=0 \\
& \alpha\left(1-3 q_{3}\right)-2 a_{5}=0 .
\end{aligned}
$$

Using (3.24) and (3.25), we obtain an $\alpha$-independent equation

$$
a_{1}\left(1-3 q_{3}\right)-a_{5}\left(q_{2}-q_{1}\right)=0,
$$

it is equivalent to

$$
\begin{aligned}
F\left(a_{1}, a_{5}\right) & =\int_{|x|=1}\left[a_{1}\left(1-3 x_{3}^{2}\right)-a_{5}\left(x_{2}^{2}-x_{1}^{2}\right)\right] e^{-a_{1}\left(x_{1}^{2}-x_{2}^{2}\right)-a_{5}\left(x_{3}^{2}-1 / 3\right)} d x \\
& =2 \int_{0}^{1} \int_{0}^{2 \pi}\left[a_{1}\left(1-3 z^{2}\right)-a_{5}\left(z^{2}-1\right) \cos 2 \varphi\right] e^{a_{1}\left(z^{2}-1\right) \cos 2 \varphi+a_{5}\left(1 / 3-z^{2}\right)} d \varphi d z \\
& =0 .
\end{aligned}
$$

From (3.27) it is straightforward to see that

$$
F\left(0, a_{5}\right)=0, \quad F\left(a_{5}, a_{5}\right)=0, \quad F\left(-a_{5}, a_{5}\right)=0 \quad \forall a_{5} \in \mathbb{R} .
$$

The rest of the proof boils down to establishing the elementary fact that there are no other zeros of the function $F\left(a_{1}, a_{5}\right)$ besides $a_{1}=0, \pm a_{5}$, whose contour lines are depicted in Figure 3.1. We do this by restricting to a specific ordering among the $q$ 's, namely $q_{3}<q_{1}<q_{2}$, which corresponds to the region where $0<a_{1}<a_{5}$. We now 


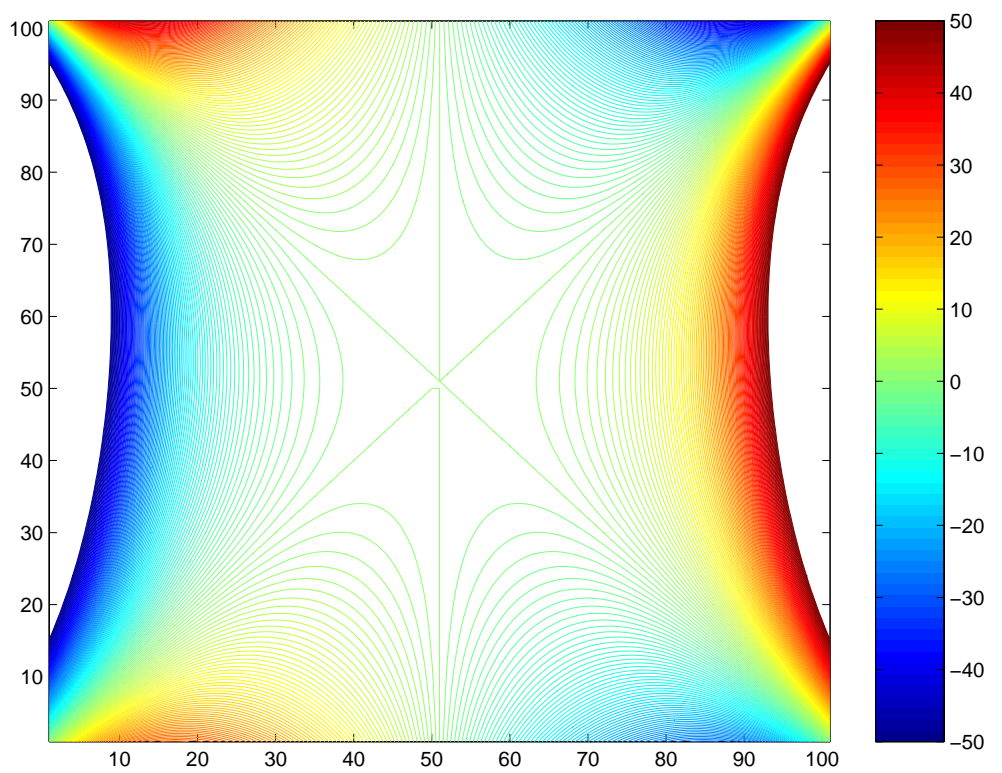

FIG. 3.1. The contour lines of function $F\left(a_{1}, a_{5}\right)$.

demonstrate $F\left(a_{1}, a_{5}\right) \neq 0$ in this region by checking the sign of $F$ along all rays $a_{5}=r a_{1}$ for $r \in(1, \infty)$. Along each ray we define

$$
\begin{aligned}
G\left(a_{1}, r a_{1}\right) & =\frac{1}{2} e^{2 a_{1} r / 3} F\left(a_{1}, a_{1} r\right) \\
& =\int_{0}^{1} \int_{0}^{2 \pi} a_{1}\left[\left(1-3 z^{2}\right)-r\left(z^{2}-1\right) \cos 2 \varphi\right] e^{a_{1}\left[\left(z^{2}-1\right) \cos 2 \varphi+r\left(1-z^{2}\right)\right]} d \varphi d z \\
& =a_{1} \sum_{n=0}^{\infty} C_{n}(r) a_{1}^{n} / n !
\end{aligned}
$$

where

$$
C_{n}(r)=\int_{0}^{1} \int_{0}^{2 \pi}\left[\left(1-3 z^{2}\right)-r\left(z^{2}-1\right) \cos 2 \varphi\right]\left[\left(z^{2}-1\right) \cos 2 \varphi+r\left(1-z^{2}\right)\right]^{n} d \varphi d z .
$$

Here the analyticity of $G$ in $a_{1}$ has been used to validate the Taylor expansion.

Set

$$
\sigma_{n}=\int_{0}^{1}\left(z^{2}-1\right)^{n} d z, \quad S_{n}(r)=\int_{0}^{2 \pi}(\cos 2 \varphi-r)^{n} d \varphi
$$

for which the recursion relations can be obtained

$$
\begin{aligned}
\sigma_{n+1} & =-\frac{2 n+2}{2 n+3} \sigma_{n}, \\
S_{n+1}(r) & =-\frac{2 n+1}{n+1} r S_{n}(r)+\frac{n}{n+1}\left(1-r^{2}\right) S_{n-1}(r) .
\end{aligned}
$$


Using these recursion relations, we have $C_{0}(r)=C_{1}(r)=0$ and

$$
\begin{aligned}
C_{n}(r) & =-r \sigma_{n+1} S_{n+1}(r)-\left(r^{2}+3\right) \sigma_{n+1} S_{n}(r)-2 \sigma_{n} S_{n}(r) \\
& =\frac{2 n}{2 n+3}\left(1-r^{2}\right)\left[S_{n}(r)+r S_{n-1}(r)\right] \sigma_{n}, \quad n \geq 2 .
\end{aligned}
$$

Noting that

$$
S_{n}(r)+r S_{n-1}(r)=\int_{0}^{2 \pi}(\cos 2 \varphi-r)^{n-1} \cos 2 \varphi d \varphi
$$

and

$$
(-1)^{n}\left[S_{n}(r)+r S_{n-1}(r)\right]>0 \quad n \geq 2, \quad(-1)^{n} \sigma_{n}>0,
$$

we obtain $C_{n}(r)<0$ for $r>1$ and $n \geq 2$. It implies that $G\left(a_{1}, a_{1} r\right) \neq 0$ for any $r>1$ and $a_{1}>0$, which enables us to conclude that $F\left(a_{1}, a_{5}\right) \neq 0$ for $0<a_{1}<a_{5}$.

The fact that there are only three zeros of $F, a_{1} \in\left\{0, \pm a_{5}\right\}$, when interpreted in terms of $q^{\prime}$ s is equivalent to $q_{i}=q_{j}, i \neq j$. Without loss of generality, we assume $q_{1}=q_{2}$, that is $a_{1}=0$. This when applied to (3.23) shows that $a_{5} Y_{5}$ is the only candidate solution to (3.10), where $a_{5}$ is an arbitrary constant. All other solutions of $V$ must be in the form of $a_{5} Y_{5}(T x)$, where $T$ is an arbitrary rotation in $\mathbb{R}^{3}$, here again we used the Lemma 3.3. Up to a scaling, we construct a family of solutions to (3.8) from $V$,

$$
\bar{U}(x)=\frac{2 \alpha}{3}+\eta\left(x_{3}^{2}-\frac{1}{3}\right)=\frac{2 \alpha}{3}-\eta\left(|x \times \bar{y}|^{2}-\frac{2}{3}\right), \quad \forall \eta \in \mathbb{R},
$$

where $\bar{y}=(0,0,1) \in \mathbb{S}^{2}$. Therefore, all solutions of $(3.8)$ are

$$
U(x)=\bar{U}(T x)=\frac{2 \alpha}{3}-\eta\left(|T x \times \bar{y}|^{2}-\frac{2}{3}\right)=\frac{2 \alpha}{3}-\eta\left(|x \times y|^{2}-\frac{2}{3}\right),
$$

where $y=T^{*} \bar{y} \in \mathbb{S}^{2}$.

The axial symmetry of $U$ when combined with Lemma 3.1 implies the axial symmetry of equilibrium solutions of the Doi-Onsager equation (3.1) with the Maier-Saupe interaction potential. Theorem 1.1 follows. We now proceed to complete the proof of Theorem 1.2.

LEMma 3.5. The solutions of (3.1) can be expressed by

$$
f=k e^{-\eta(x \cdot y)^{2}},
$$

where $\eta, k$ depend on $\alpha$ through the relations:

$$
\begin{aligned}
& \frac{3 e^{-\eta}}{\int_{0}^{1} e^{-\eta z^{2}} d z}-\left(3-2 \eta+\frac{4 \eta^{2}}{\alpha}\right)=0, \\
& k=\left[4 \pi \int_{0}^{1} e^{-\eta z^{2}} d z\right]^{-1} .
\end{aligned}
$$

Proof. From Lemma 3.1 and 3.4, we have

$$
f=C e^{-U}=C e^{-2 \alpha / 3} e^{\eta\left(|x \times y|^{2}-2 / 3\right)}=C e^{-2 \alpha / 3} e^{\eta / 3} e^{-\eta(x \cdot y)^{2}} .
$$


Since both $C$ and $\eta$ are to be determined, we change $C e^{-2 \alpha / 3} e^{\eta / 3}$ to $k$ and obtain (3.29). From the proof of Lemma 3.4 we know $y=T \bar{y}$, where $\bar{y}=(0,0,1)$. Without loss of generality we focus on the case $y=\bar{y}$. Other solutions are just $U(T x, \bar{y})=U(x, T \bar{y})$. The normalization (3.3) with (3.29) gives

$$
k \int_{|x|=1} e^{-\eta x_{3}^{2}} d x-1=0
$$

which proves (3.31) for

$$
4 \pi k \int_{0}^{1} e^{-\eta z^{2}} d z=1
$$

Using the nonlocal constraint (3.2) we obtain the following relation

$$
\frac{2 \alpha}{3}-\eta\left(\frac{1}{3}-x_{3}^{2}\right)=k \alpha \int_{\left|x^{\prime}\right|=1}\left[1-\left(x \cdot x^{\prime}\right)^{2}\right] e^{-\eta x_{3}^{\prime 2}} d x^{\prime}
$$

which combined with (3.33) yields

$$
1-k \int_{\left|x^{\prime}\right|=1}\left(x \cdot x^{\prime}\right)^{2} e^{-\eta x_{3}^{\prime 2}} d x^{\prime}=-\frac{\eta}{\alpha}\left(\frac{1}{3}-x_{3}^{2}\right)+\frac{2}{3} .
$$

Now we proceed to simplify (3.35). Using the polar coordinate (3.11), we have

$$
\begin{aligned}
\left(x \cdot x^{\prime}\right)^{2}= & {\left[\cos \theta \cos \theta^{\prime}+\cos \left(\varphi-\varphi^{\prime}\right) \sin \theta \sin \theta^{\prime}\right]^{2} } \\
= & \cos ^{2} \theta \cos ^{2} \theta^{\prime}+\cos ^{2}\left(\varphi-\varphi^{\prime}\right) \sin ^{2} \theta \sin ^{2} \theta^{\prime}+\frac{1}{2} \cos \left(\varphi-\varphi^{\prime}\right) \sin 2 \theta \sin 2 \theta^{\prime} \\
= & \frac{1}{2}\left[1-\cos ^{2} \theta-\cos ^{2} \theta^{\prime}+3 \cos ^{2} \theta \cos ^{2} \theta^{\prime}+\cos 2\left(\varphi-\varphi^{\prime}\right) \sin ^{2} \theta \sin ^{2} \theta^{\prime}\right. \\
& \left.+\cos \left(\varphi-\varphi^{\prime}\right) \sin 2 \theta \sin 2 \theta^{\prime}\right] .
\end{aligned}
$$

This fact combined with (3.35) yields

$$
\begin{aligned}
0= & \frac{1}{3}-\frac{k}{2} \int_{0}^{2 \pi} \int_{0}^{\pi}\left[1-\cos ^{2} \theta-\cos ^{2} \theta^{\prime}+3 \cos ^{2} \theta \cos ^{2} \theta^{\prime}\right] e^{-\eta \cos ^{2} \theta^{\prime}} \sin \theta^{\prime} d \theta^{\prime} d \varphi^{\prime} \\
& +\frac{\eta}{\alpha}\left(\frac{1}{3}-\cos ^{2} \theta\right) \\
= & -\frac{1 / 3-\cos ^{2} \theta}{2}+\frac{3 k\left(1 / 3-\cos ^{2} \theta\right)}{2} \int_{0}^{2 \pi} \int_{0}^{\pi} \cos ^{2} \theta^{\prime} e^{-\eta \cos ^{2} \theta^{\prime}} \sin \theta^{\prime} d \theta^{\prime} d \varphi^{\prime} \\
& +\frac{\eta}{\alpha}\left(\frac{1}{3}-\cos ^{2} \theta\right)
\end{aligned}
$$

This implies

$$
k \int_{0}^{2 \pi} \int_{0}^{\pi} \cos ^{2} \theta^{\prime} e^{-\eta \cos ^{2} \theta^{\prime}} \sin \theta^{\prime} d \theta^{\prime} d \varphi^{\prime}-\frac{1}{3}+\frac{2 \eta}{3 \alpha}=0,
$$

which can be further simplified as

$$
4 \pi k \int_{0}^{1} z^{2} e^{-\eta z^{2}} d z=\frac{1}{3}-\frac{2 \eta}{3 \alpha} .
$$


(3.37) divided by (3.34) shows that $\alpha$ and $\eta$ satisfy

$$
\int_{0}^{1} z^{2} e^{-\eta z^{2}} d z=\frac{\alpha-2 \eta}{3 \alpha} \int_{0}^{1} e^{-\eta z^{2}} d z
$$

Integration by part yields

$$
\begin{aligned}
\int_{0}^{1} e^{-\eta z^{2}} d z & =e^{-\eta}+2 \eta \int_{0}^{1} z^{2} e^{-\eta z^{2}} d z \\
& =e^{-\eta}+2 \eta \frac{\alpha-2 \eta}{3 \alpha} \int_{0}^{1} e^{-\eta z^{2}} d z
\end{aligned}
$$

This leads to the relation (3.30).

From Lemma 3.5 we see that in order to determine the number of solutions of (3.1), it suffices to determine the number of zeros of $B(\eta, \alpha)$ in term of $\alpha$, where

$$
B(\eta, \alpha) \triangleq \frac{3 e^{-\eta}}{\int_{0}^{1} e^{-\eta z^{2}} d z}-\left(3-2 \eta+\frac{4 \eta^{2}}{\alpha}\right) .
$$

LEMma 3.6. The number of zeros of $B(\eta, \alpha)$ is determined by the intensity $\alpha$ as follows:

(i). If $\alpha>7.5, B(\eta, \alpha)$ has three zeros $\eta_{1}^{*}<0, \eta_{2}^{*}>0$ and $\eta_{0}=0$.

(ii). If $\alpha=7.5, B(\eta, \alpha)$ has two zeros $\eta_{1}^{*}<0$ and $\eta_{0}=0$.

(iii). There exists an $\alpha^{*} \in(20 / 3,7.5)$ such that $B(\eta, \alpha)$ has three zeros $\eta_{1}^{*}<0, \eta_{2}^{*}<$ 0 and $\eta_{0}=0$ for $\alpha^{*}<\alpha<7.5$.

(iv). If $\alpha=\alpha^{*}, B(\eta, \alpha)$ has two zeros $\eta_{1}^{*}<0$ and $\eta_{0}=0$.

(v). If $0<\alpha<\alpha^{*}, B(\eta, \alpha)$ has one zero $\eta_{0}=0$.

Proof. We will complete the proof in five steps.

Step 1. We first show that

$$
B( \pm M, \alpha)<0 \quad \text { for } M \gg 1 \text {. }
$$

Note that, for $\eta>0$, the mean value theorem gives $3 e^{-\eta} / \int_{0}^{1} e^{-\eta z^{2}} d z=3 e^{-\eta\left(1-\gamma^{2}\right)} \in$ $(0,3)$ for some $\gamma \in(0,1)$. For $\eta<0$, we have from $(3.39)$

$$
e^{-\eta}=\int_{0}^{1} e^{-\eta z^{2}} d z-2 \eta \int_{0}^{1} z^{2} e^{-\eta z^{2}} d z \leq(1-2 \eta) \int_{0}^{1} e^{-\eta z^{2}} d z .
$$

This shows $0<\frac{3 e^{-\eta}}{\int_{0}^{1} e^{-\eta z^{2}} d z} \leq 3(1-2 \eta)$. Thus for large $|\eta| \gg 1, B$ is determined by the quadratic term $-4 \eta^{2} / \alpha$. (3.41) follows.

Step 2. If $\alpha>7.5$ we will show that $B(\eta, \alpha)$ has at least two zeros $\eta_{1}<0$, and $\eta_{2}>0$. We know that $\eta=0$ is always a zero since $B(0, \alpha)=0$. Moreover, using (3.38) we have

$$
\begin{aligned}
B_{\eta}(\eta, \alpha) & =\frac{-3 e^{-\eta} \int_{0}^{1} e^{-\eta z^{2}} d z+3 e^{-\eta} \int_{0}^{1} z^{2} e^{-\eta z^{2}} d z}{\left(\int_{0}^{1} e^{-\eta z^{2}} d z\right)^{2}}-\left(-2+\frac{8 \eta}{\alpha}\right) \\
& =\frac{3 e^{-\eta}}{\int_{0}^{1} e^{-\eta z^{2}} d z}\left(-1+\frac{\alpha-2 \eta}{3 \alpha}\right)+2\left(1-\frac{4 \eta}{\alpha}\right) \\
& =\frac{-2 e^{-\eta}}{\int_{0}^{1} e^{-\eta z^{2}} d z}\left(1+\frac{\eta}{\alpha}\right)+2\left(1-\frac{4 \eta}{\alpha}\right) .
\end{aligned}
$$


This yields $B_{\eta}(0, \alpha)=0$. Therefore, $\eta=0$ is a double zero of $B$ for every $\alpha$. Hence the local shape of $B$ needs to be determined by $B_{\eta \eta}(0, \alpha)$. A further calculation from (3.40) gives

$$
B_{\eta \eta}(0, \alpha)=\frac{4}{3 \alpha}\left(\alpha-\frac{15}{2}\right)
$$

Thus $B_{\eta \eta}(0, \alpha)>0$ for $\alpha>7.5 . \quad B$ is locally convex near $\eta=0$. This together with (3.41) implies that there exist at least two zeros $\eta_{1}^{*}<0$ and $\eta_{2}^{*}>0$ besides $\eta=0$. Now we assume $\eta^{*}$ is a zero of $B$, i.e.,

$$
\frac{3 e^{-\eta^{*}}}{\int_{0}^{1} e^{-\eta^{*} z^{2}} d z}=3-2 \eta^{*}+\frac{4 \eta^{* 2}}{\alpha} .
$$

This inserted into (3.42) gives

$$
\begin{aligned}
B_{\eta}\left(\eta^{*}, \alpha\right) & =-\frac{8 \eta^{*}}{3 \alpha^{2}}\left(\eta^{* 2}+\frac{\eta^{*} \alpha}{2}+\frac{\alpha}{2}\left(\frac{15}{2}-\alpha\right)\right) \\
& =\left\{\begin{array}{lll}
-\frac{8 \eta^{*}}{3 \alpha^{2}}\left(\eta^{*}-\bar{\eta}_{1}\right)\left(\eta^{*}-\bar{\eta}_{2}\right), & \text { if } & \alpha>20 / 3, \\
-\frac{8 \eta^{*}}{3 \alpha^{2}}\left(\eta^{*}+\frac{\alpha}{4}\right)^{2}, & \text { if } & \alpha=20 / 3, \\
-\frac{8 \eta^{*}}{3 \alpha^{2}}\left[\left(\eta^{*}+\frac{\alpha}{4}\right)^{2}+\frac{15 \alpha}{4}\left(1-\frac{3 \alpha}{20}\right)\right], & \text { if } & \alpha<20 / 3,
\end{array}\right.
\end{aligned}
$$

where

$$
\bar{\eta}_{1}=-\frac{\alpha}{4}\left(1+3 \sqrt{1-\frac{20}{3 \alpha}}\right), \quad \bar{\eta}_{2}=-\frac{\alpha}{4}\left(1-3 \sqrt{1-\frac{20}{3 \alpha}}\right)
$$

From (3.46), we see that $\eta_{1}^{*}<\bar{\eta}_{1}<-\alpha / 2$, and $\eta_{2}^{*}>\bar{\eta}_{2}>0$.

Step 3. We now show that $B(\eta, \alpha)$ has at most two zeros besides 0 for $\alpha>7.5$. From $(3.46), B_{\eta}\left(\eta^{*}, \alpha\right)<0$ for $\eta^{*} \in\left(\bar{\eta}_{2}, \infty\right)$. This implies that there is at most one zero of $B$ in $(0, \infty)$. Otherwise $B_{\eta}\left(\eta^{*}, \alpha\right)$ has to be negative at another zero. Similarly, there exists at most one $\eta^{*} \in\left(-\infty, \bar{\eta}_{1}\right)$. The claim in (i) is thus proved.

Step 4. We now consider the case $\alpha=7.5$, for which we show that there exist two zeros $\eta_{1}^{*}<0$ and 0 . In this case, $B_{\eta}(0, \alpha)=B_{\eta \eta}(0, \alpha)=0$. In order to see the local shape of $B$ at $\eta=0$, we calculate

$$
B_{\eta \eta \eta}(0, \alpha)=-\frac{16}{45}<0 .
$$

This means

$$
\eta B(\eta, \alpha)<0 \quad \text { for } \quad|\eta| \ll 1
$$

(3.45) with $\alpha=7.5$ gives

$$
B_{\eta}\left(\eta^{*}, \alpha\right)=-\frac{8 \eta^{* 2}}{3 \alpha^{2}}\left(\eta^{*}+\frac{\alpha}{2}\right)
$$

where $\eta^{*}$ is assumed to be a zero of $B$. The local behavior implied from (3.49) and the negative sign of $B_{\eta}\left(\eta^{*}, \alpha\right)$ for $\eta^{*}>0$ shows that no zero of $B$ exists in $(0, \infty)$. On the other hand, (3.49), together with $B(-M, \alpha)<0$ shows that there exists at least one 
zero in $(-\infty, 0)$. We denote it by $\eta_{1}^{*}$. By $(3.50)$, we know $\eta_{1}^{*}<-\alpha / 2$ and $B_{\eta}\left(\eta_{1}^{*}, \alpha\right)>0$. Now we claim $\eta_{1}^{*}$ is a unique zero of $B$ in $(-\infty, 0)$. Otherwise, there should appear at least two more zeros in $(-\infty, 0)$, which is not allowed by $(3.50)$. This proves (ii).

Step 5. As argued in step 4 , we can show that $B(\eta, \alpha)$ has no zero in $(0, \infty)$ for $\alpha<7.5$. Moreover, if $\alpha \leq 20 / 3, B(\eta, \alpha)$ even has no zero in $(-\infty, 0)$. This is ensured by the fact $B(-M, \alpha)<0$ and the sign constrained by (3.46), as argued in step 4 .

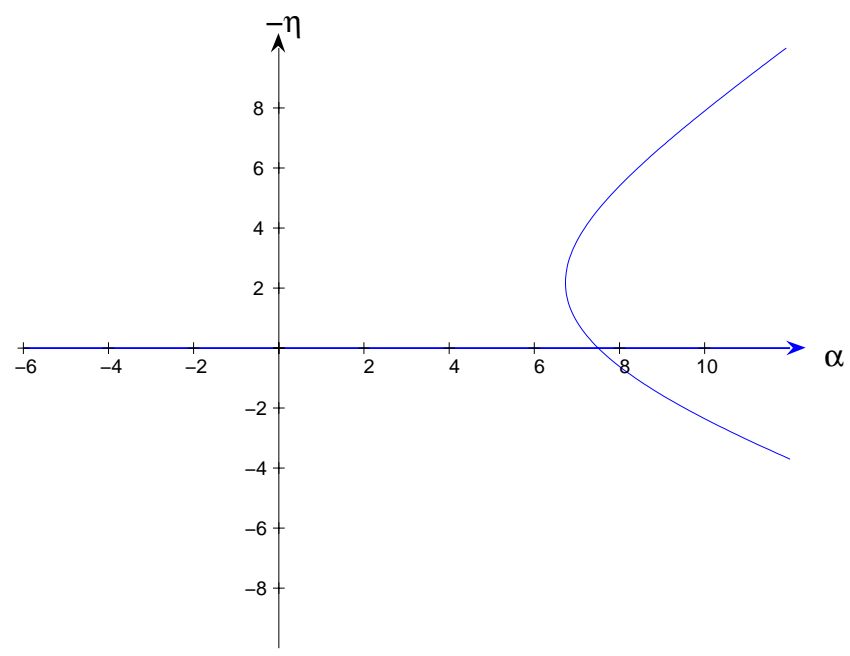

FIG. 3.2. The relation between $\alpha$ and $\eta$.

In order to identify the second critical value $\alpha^{*} \in(20 / 3,7.5)$, we need to use a continuity argument. First for $7.5-\delta<\alpha<7.5, \delta>0$ small, there are two zeros $\eta_{1}^{*}, \eta_{2}^{*}<$ 0 of $B$. In fact for this range of $\alpha, B_{\eta \eta}(0, \alpha)<0 . B(\eta, \alpha)$ is locally concave near $\eta=0$. We also know that $B(\eta, 7.5)>0$ for $\eta \in(-\delta, 0)$. This together with $B(-M, \alpha)<0$ shows that there are two zeros of $B$ in $(-\infty, 0)$. From $(3.46)$,

$$
\eta_{1}^{*} \leq \overline{\eta_{1}}, \quad \overline{\eta_{1}} \leq \eta_{2}^{*}<\overline{\eta_{2}} .
$$

On the other hand for $\alpha \leq 20 / 3, B(\eta, \alpha)<0$ for $\eta \in(-\infty, 0)$. By continuity of $B$ in $\alpha$, as $\alpha$ decreases in $(20 / 3,7.5)$, two zeros of $B$ will become closer and get together at a point $\alpha=\alpha^{*}$ :

$$
\eta_{1}^{*}=\overline{\eta_{1}}\left(\alpha^{*}\right)=\eta_{2}^{*}
$$

at which $B\left(\overline{\eta_{1}}\left(\alpha^{*}\right), \alpha^{*}\right)=B_{\eta}\left(\overline{\eta_{1}}\left(\alpha^{*}\right), \alpha^{*}\right)=0$. That is, when $\alpha=\alpha^{*}, \overline{\eta_{1}}\left(\alpha^{*}\right)$ is also a double zero. For $20 / 3<\alpha<\alpha^{*}, B(\eta, \alpha)$ has no zero in $(-\infty, 0)$ either. Thus $\alpha^{*}$, instead of $20 / 3$, is indeed a critical value. These all together finish the proof of (iii)-(v).

We now conclude this paper by giving a formula for $\alpha^{*}$. Using (3.38), we have

$$
\alpha=\frac{2 \eta \int_{0}^{1} e^{-\eta z^{2}} d z}{\int_{0}^{1}\left(1-3 z^{2}\right) e^{-\eta z^{2}} d z}=\frac{\int_{0}^{1} e^{-\eta z^{2}} d z}{\int_{0}^{1}\left(z^{2}-z^{4}\right) e^{-\eta z^{2}} d z},
$$


from which a relation between $\alpha$ and $-\eta$, besides the isotropic case $\eta=0$, can be visualized in Figure 3.2. The second critical intensity $\alpha^{*}$ can be expressed as

$$
\alpha^{*}=\min _{\eta} \frac{\int_{0}^{1} e^{-\eta z^{2}} d z}{\int_{0}^{1}\left(z^{2}-z^{4}\right) e^{-\eta z^{2}} d z},
$$

which is about $\alpha^{*} \approx 6.731393$ from our numerical calculation.

Acknowledgments. The authors are very grateful to Professors Weinan E, Chun Liu and Qi Wang for their helpful discussions. Hailiang Liu's research is supported in part by the New Collaborative Research Grant of Ames Lab, the U.S. Department of Energy. Hui Zhang's research is supported by National Science Foundation of China 10401008. Pingwen Zhang's research is partially supported by the special funds for Major State Research Projects G1999032804 and National Science Foundation of China for Distinguished Young Scholars 10225103.

\section{REFERENCES}

[1] P. Constantin, I. Kevrekidis and E. S. Titi, Asymptotic states of a Smoluchowski equation, Arch. Rat. Mech. Anal., 174, 365-384, 2004.

[2] P. Constantin, I. Kevrekidis and E. S. Titi, Remarks on a Smoluchowski equation, Discrete and Continuous Dynamical Systems, 11, 101-112, 2004.

[3] P. Constantin and J. Vukadinovic, Note on the number of steady states for a 2D Smoluchowski equation, Nonlinearity, 18, 441-443, 2005.

[4] M. Doi and S. F. Edwards, The Theory of Polymer Dynamics, Oxford University Press, 1986.

[5] S. Engelberg, H. L. Liu and E. Tadmor, Critical thresholds in Euler-Poisson equations, Indiana Univ. Math. J., 50, 109-157, 2001.

[6] I. Fatkullin and V. Slastikov, A note on the Onsager model of nematic phase transitions, Comm. Math. Sci., 3, 1, 21-26, 2005.

[7] I. Fatkullin and V. Slastikov, Critical points of the Onsager functional on a sphere, preprint.

[8] V. Faraoni, M. Grosso, S. Crescitelli and P. L. Maffettone, The rigid-rod model for nematic polymers: an analysis of the shear flow problem, J. Rheol., 43, 829-843, 1999.

[9] M. G. Forest, R. Zhou and Q. Wang, Full-tensor alignment criteria for sheared nematic polymers, J. Rheol., 47, 105-127, 2003.

[10] P. G. de Gennes and J. Prost, The Physics of Liquid Crystals, 2nd edn, Oxford Science Publications, 1993.

[11] R. Jordan, D. Kinderlehrer and F. Otto, The variational formulation of the Fokker-Planck equation, SIAM J. Math. Anal, 29, 1-17, 1998.

[12] H. L. Liu and E. Tadmor, Critical thresholds in a convolution model for nonlinear conservation laws, SIAM J. Math. Anal., 33, 930-945, 2002.

[13] C. Luo, H. Zhang and P.W. Zhang, The structure of equilibrium solutions of the onedimensional Doi equation, Nonlinearity, 18, 379-389, 2005.

[14] P. L. Maffettone and S. Crescitelli, Bifurcation analysis of a molecular model for nematic polymers in shear flows, J. Non-Newtonian Fluid Mech., 59, 73-91, 1995.

[15] G. Marrucci and P. L. Maffettone, Description of the liquid crystalline phase of rodlike polymers at high shear rates, Macromolecules, 22, 4076-4082, 1989.

[16] L. Onsager, The effects of shape on the interaction of colloidal particles, Ann. N. Y. Acad. Sci., 51, 627-659, 1949. 